\title{
Stakeholder Insights Into a Systems-Based Suicide Prevention Program Implemented in Regional and Rural Tasmanian Communities
}

Laura Grattidge ( $\square$ laura.grattidge@utas.edu.au )

University of Tasmania

Jonathan Mond

University of Tasmania

Stuart Auckland

University of Tasmania

Terry Purton

University of Tasmania

David Lees

University of Tasmania

Research Article

Keywords: stakeholders, Prevention

Posted Date: November 29th, 2021

DOI: https://doi.org/10.21203/rs.3.rs-1045269/v1

License: (c) (1) This work is licensed under a Creative Commons Attribution 4.0 International License.

Read Full License 


\section{Abstract}

Purpose: Emerging evidence indicates that systems-based suicide prevention programs can help optimise suicide prevention activities, with the National Suicide Prevention Trial using these approaches in regional and community contexts throughout Australia. The Tasmanian arm of the Trial adopted the LifeSpan systems framework to deliver suicide prevention activities across three distinct geographical areas, focusing on high-risk populations of men aged 40-64 and people 65 and over. The University of Tasmania's Centre for Rural Health undertook a local-level evaluation of the Trial in Tasmania.

Aims: To explore key stakeholder perceptions of the implementation of a systems-based suicide prevention program in regional and rural communities in Tasmania, Australia.

Method: Focus groups and interviews with 46 participants, comprising Working Group members $(n=25)$, Tasmania's Primary Health Network employees $(n=7)$, and other key stakeholders $(n=14)$, with the majority (53.3\%) reporting a lived experience of suicide. Thematic analysis was used to explore data and study aims.

Results: Key themes centred on how the National Suicide Prevention Trial was understood and established in Tasmania; Working Group governance structures and processes; communication and engagement processes; reaching priority population groups; the LifeSpan model and activity development; and the effectiveness and sustainability of activities.

Discussion: Findings showed communities were wary of suicide and wanted to engage to take action and the Trial provided the resources and coordination to do so. Perceived limitations implementing the Trial included varied involvement of key stakeholders, and lack of role clarity within Working Groups. Barriers delivering activities to the priority population groups suggested a strict adherence to the Lifespan model was challenging. Working Groups embraced a pragmatic approach, preferring activities that best utilised available capital and resources to meet perceived needs within communities. While a focus on effectiveness and sustainability of activities was seen as important, barriers at the community-level, i.e. nobody to run them, hindered these efforts. Analysis of stakeholder perceptions provides crucial insights for guiding future community-based suicide prevention efforts in regional and rural areas, and with highrisk groups.

\section{Introduction}

Suicide is a major public health problem, with 3,139 people in Australia dying by suicide in 2020[1]. This is a slight decrease from 3,318 in 2019 [2] (12.9 to 12.1 deaths per 100,000 people), however still an increase from 10.7 in 2009[3]. There are further increases predicted in the wake of COVID-19, with impacts extending across all systems and societies [3-5]. Recognising the need for new approaches to suicide prevention, the Australian Government, in 2016, initiated the National Suicide Prevention Trial (the Trial), to utilise systems-based approaches and the breadth of resources available at the community-level [6]. 
Twelve sites across Australia were selected through the Trial, with Primary Health Networks receiving funding over four years to coordinate efforts within their local communities (Figure 1). Site selection was based on a number of community-level factors, including local suicide rates and capacity to implement initiatives. Trial goals included reaching at-risk groups, and trialling systems-based approaches to suicide prevention[6, 7]. Australian trials of systems approaches to suicide prevention are in their infancy[8-11], with recent trials in Europe, the US, and Asia[12-15] providing preliminary support, however highlighting a need for further research. Systems approaches are based on the premise that suicide and its prevention are situated within complex, multilevel systems, and the occurrence and adverse impacts of suicide can be reduced if a range of strategies are simultaneously implemented across these systems $[9,10,16]$. Importantly, the multifactorial nature of systems approaches means they are also practically complex, and due to their recency in Australia, evidence as to their utility in areas of regional, rural and remote areas of the country remains limited[17].

Tasmania was selected as one of the 12 trial sites[18]. Australia's only island state is home to approximately 540,000 people and has a rate of suicide $(15.9$ per 100,000$)$, second only to the Northern Territory (20.4)[1]. Under the Modified Monash Model remoteness classification, the Tasmanian capital city, Hobart, is classified as an MM2 rating (regional centre), as too is the city of Launceston, with the rest of the state, classified between MM2 to MM5 (regional centres to small rural towns). Making up the Tasmanian trial site were the Local Government Areas of Launceston in Northern Tasmania (regional centre), Break O'Day a local government area (LGA) on the North-East coast (comprising small and large rural towns), and the North-West Coast LGAS of Devonport, the Central Coast and Burnie (large rural towns)[19]. Site selection was based on consideration of existing physical and social infrastructure, resources and suicide prevention activities, community suicide risk factor profiles, and local capacity to be involved[7]. Working Groups were convened for each of the trial site locations to plan and undertake activities, and were comprised of a funded coordinator, chair, host organisation manager, local community members, and representatives from service organisations and Tasmania's Primary Health Network[7, 18].

The priority population groups for Tasmania - chosen by the Tasmanian Suicide Prevention Trial Advisory Group (the program's overarching governing committee) in consultation with stakeholders were men aged 40 to 64 years and people 65 years and over. The Advisory Group also had responsibility for the selection of the Black Dog Institute's LifeSpan framework[7, 9] as the systems-based approach to guide activities. The Lifespan framework combines nine strategies, for which available evidence was deemed strongest at the time of framework development, and aims to build community capacity to better support people facing suicidal crisis[9, 20] (Figure 2).

The University of Tasmania's Centre for Rural Health were engaged to evaluate the Tasmanian trial site as a process-oriented evaluation, aimed to supplement a more outcome-oriented, national-level evaluation conducted by the University of Melbourne. A Participatory Action Research approach was employed as a 
reflective process of collaborating with Working Groups in the design and conduct of evaluation activities $[7,18]$. This approach aligns to the literature highlighting the importance of taking into account both a collaborative approach and public opinion when developing, refining, implementing, and evaluating health promotion programs[21, 22].

The literature evaluating suicide prevention initiatives in Australia has largely focused on the utility of specific training or intervention programs or isolated strategies, rather than the feasibility of broad or synergistic (systems-based) approaches. Further, evidence evaluating the effectiveness of community-led programs in regional, rural and remote regions of Australia is particularly limited[23-27]. The study aims to explore the views of key stakeholders involved with the trial in Tasmania, to elucidate perceptions surrounding the development and implementation of suicide prevention activities using a systems-based approach. Findings will inform the development and implementation of future public health interventions, including those for suicide prevention, particularly when such interventions are in regional/rural areas, focused on at-risk population groups, and are using a systems-based approach.

\section{Methodology}

\section{Design}

As previously mentioned, a Participatory Action Research approach was utilised throughout the evaluation, as a reciprocal process of action and reflection, to guide the design and conduct of evaluation activities $[7,18]$. Interviews and focus groups were employed, as qualitative methods that are commonly used in suicidology, to provide explanations and understandings and to give voice to participants, and in particular people with lived experience[28, 29].

\section{Participants}

Participants comprised 46 people involved with the trial in Tasmania, including Working Group members $(n=25)$ across all three site locations (North-West Coast, Launceston and Break O'Day (Figure 3), project staff at Tasmania's Primary Health Network $(n=7)$, and several external stakeholders, including the national evaluation team, peak suicide prevention body representatives, Advisory Group members, and members of the Tasmanian Suicide Prevention Community Network $(n=14)$.

Participant ages ranged from 28 to 75 years (median=53 years, $S D=11.7)$. Just over half identified as female (51.1\%) and the majority (53.3\%) reported a lived experience of suicide, as defined by Roses in the Ocean[30].

\section{Data collection and analysis}

Data were collected between January and March 2020 across the three trial site locations. Participants provided informed consent prior to participation. Focus groups and interviews were guided by semistructured questions relating to key governance processes and structures, including decision making, 
partnerships and Working Group functions, and development of activities. Focus groups and interviews lasted for an average of 59 minutes. Data were collected until data saturation was reached[31]. Sessions were audio-recorded and transcribed verbatim, and participants' names were replaced with ID numbers for confidentiality. Initial data analysis was guided by the six phases of thematic analysis (Braun, 2006) and data were stored, coded, classified, and sorted using NVivo software (QSR International Pty Ltd, version 10,2014). Data integrity and analysis were confirmed by a second member of the research team and, where required, incongruent results were managed by discussion to reach consensus.

All methods were carried out in accordance with relevant guidelines and regulations and experimental protocols that were approved by the Human Research Ethics Committee (Tasmania) Network (H0017793). Informed consent was obtained from all participants.

\section{Results}

Six key themes were identified from focus group and interview data (Figure 4.), which will be discussed in the following section.

Participants are represented as either a Working Group member (WG1, WG2 ...), Tasmania's Primary Health Network employee (PHN1, PHN2 ...), or external stakeholder (E1, E2 ...), to ensure confidentiality.

\section{Understanding and establishing the trial in Tasmania}

The establishment of the trial was seen to be dependent on early decisions made by the Advisory Group, who, in their role as higher-level project governance representatives, played a key role as major decision makers throughout the Trial.

- ...they [the Advisory Group] had an integral and fundamental role to play in the design of the trial. You know, identifying our target populations, where the trial was geographically situated, and in approving some of the initiatives. (PHN6)

Having host organisations already working in suicide prevention was thought to help with the establishment of Working Groups, through existing links to local communities, businesses, and service providers.

- ...having had the mental health action group on the ground and already involved in that space gave us a little bit of a head start, because there were already some layers that had been put down to help this work. (WG10)

A voluntary and committed approach from Working Groups members, some bringing their own lived experience, was seen to support the establishment of the trial and provide motivation to overcome challenges, including bureaucratic/administrative burden. 
- The Working Group members'voluntary approach, their passion for their community to enable this to be established, to bear with the process and the bureaucracy and continue, it's a big commitment. (WG4)

Establishing the trial was seen to take time and effort and require the people with the appropriate knowledge and skill sets to sit at the table, particularly when developing feasible action plans.

“...had to engage staff, in some instances, recruit staff, inform staff, and then those staff members and the key agencies were responsible for bringing the Working Groups together and designing their action plans... for the Working Groups themselves to say, you know, have we got the right people around the table and if not, who else needs to be here? (PHN6)

From an implementation perspective, the Working Group members were seen to be more motivated to engage when they understood the Trial aims and the purpose of their involvement. It was generally understood that the Trial was looking at novel approaches to reach at-risk groups and provide resources to build local community capacity:

- ...how could we respond more effectively in terms of suicide prevention given those key target groups?...how could we develop the capacity of the community?...[the trial was] a program that was about innovation and saying we need something different than traditional health paradigms. (E1)

There was also an understanding that the Trial would be an opportunity to explore how a systems-based approach could be applied, and the capacity communities have to influence these systems using the LifeSpan framework:

- The trial is an opportunity to test the systems approach... looking at community capacity to influence systems across the board using LifeSpan. (PHN6)

The following Working Group member, reported their understanding of Trial aims as using different approaches to reach specific population groups.

- My understanding of the suicide prevention trial site is trialling different strategies and approaches to suicide prevention... a particular client demographicmen aged 45 plus and women 65 and older. (WG4)

\section{Working Group governance structures and processes}

From establishment, the role of the PHN was seen as essential to supporting the Working Groups, as described by these participants from the PHN.

- There is a lot of bureaucratic hurdles that the working groups and the Coordinators had to jump over and the [PHN] Consultant could actually support the coordinator and the Host Organisation through that process. (PHN1) 
- It was a lot of bureaucratic processes. You know, the action plans and reports and all of that and that we were able to provide that direct guidance around it. (PHN4)

Tasmania's PHN acted as a "conduit of advice", an access point or "connector" for information for the Working Group; for example, the partnership established with the Coroner's office.

- The relationship that we built with the Coroner's office, and, you know, for a Coroner's office to offer an NGO access to some of the data they allowed us to access, you know, we had to prove to them that we could manage that data in a very sound and capable way...they may need organisations like a Primary Health Network or similar to enable, that kind of conduit of advice. (PHN5)

This following Working Group member described how the PHN provided flexibility around learning along the way and showed this throughout the Trial with constant reflection and quality improvements.

- [PHN] has been quite flexible in a lot of ways because I think, to be fair, they've been limping through a lot of these things as well. They've learned okay, by trial and error they found out this is working, or this isn't. And a lot of the downs when things didn't get off the ground...they've learned that, you know, these are some of our difficulties and they've been quite flexible with that as well. (WG2)

Whilst the Trial was understood as looking at the capacity of communities, the model itself was administered through Tasmania's Primary Health Network and as described by the following participant, seen as embedded within the traditional health paradigm,

- The real purpose of it was to try and capture words on paper that can be shown to the health bureaucrats, you know, actually facilitate that whole thing from saying that this shouldn't be dominated by health model[s]. But on the same hand, you've got an organization which was [PHN], which is absolutely and utterly dominated by traditional health paradigms. (E1)

Working Group members believed additional flexibility is required with managing budgets and reconciling how funds were spent:

- In the initial stage things were quite highly directive, an unusual method...the level of hands-on by PHN has been...not one I would endorse going forward... (WG12)

- ... it's been really time-consuming as well as non-user-friendly. (WG4)

The importance of transparency around funding processes was highlighted by participants. It was expressed that those processes should be streamlined with funds easily accessible, as they can impact activity selection and decision making.

- Where you're trying to get community-made action and suicide prevention, you need to make it as easy as possible... when they need resources, they need to be readily available alongside that. (E10) 
Administrative processes were also considered overly burdensome, with levels of bureaucracy potentially impacting progress, highlighting a need to initial explore community literacy and capacity.

- It's been quite restrictive, and I've seen the admin work that had to be done. There's a lot of bureaucracy... we can see things that need to be done and we want to do them and we're not able to do them...it's not a lack of us wanting to, it's a lack of resources being given and powers-that-be holding back, for whatever reason. (WG8)

It was, however, understood that many of these governance and administrative processes were driven by contractual obligations associated with participating in the Trial.

- This is part of a national trial. And that brings with it certain obligations and ways of working and accountability... we can't just let money go out the door without knowing what it's for, the evidence for it, how it's going to be expended? (PHN6)

Working Group progress was influenced by locally available social and physical capital, with each group having a slightly different structure and membership composition, influencing activity selection and success.

- We utilised our existing organisations such as Men's Shed, Rotary events...Sporting clubs, annual dinners... we utilised those existing networks. (WG19)

Having grassroots community member involvement was seen as pivotal to Working Groups, recognising the contribution and experience of members already working in the community and a means to shift a top-down approach to bottom-up.

- ...we've become more community-focused, so that the people who are now on the group were already working in the community, making those kinds of connections...it's shifted from top-down to ground level where the work is really being done. (WG19)

The role of the coordinator was central to Trial site operations and successes, likened to a "relationship and management" role, the "anchor" connecting the trial with local community.

- It's nearly like a manager... supporting ... giving advice, information, empowering them [the Working Group]. (WG4)

The inclusion of community members with lived experience in Working Groups was also acknowledged as a powerful enabler, bringing a different perspective and much-needed energy.

- ... they had the more 'can-do' approach that really changed the energy in the room... They came up with ideas... changed the way people thought about things... how do we make this work? How do we fix it?... How do we overcome it?" (WG2) 
It was acknowledged that often Working Group membership was made up of people representing services as a requirement of their job roles, and that this may have affected the level of buy-in for these members.

- One of the things that I think was really missed in this trial as it was implemented here was actually picking up people who were genuine...Some of them had lived experience, some or everybody had some form of motivation to be involved... generally, they were picked up because they hold positions of some sort. (E1)

Given the nature of it being a trial, lack of role clarity and decision-making structures were described by Working Groups, manifesting as inactivity:

- All the Working Group meetings were just conversations, nothing really got done... We didn't think we could make those decisions because it'd have to be a group decision, but then the group didn't think that it was their responsibility to make those decisions. PHN didn't want to make those decisions because they're a funder...So I think it was conflicting...So we had all these rounds of nonconversations. (WG2)

\section{Communication and engagement processes}

Communication across the Trial site was impacted by different communication styles, and people interpreting things differently, tying in a need for clear Terms of Reference at program outset to guide expectations and responsibilities.

- There were different communication styles and things like that, so that was also challenging at times. People interpreted things differently. (WG25)

The Participatory Action Research process helped unpack the processes of the Trial, including the role of stakeholders and relationships needed.

- ... all of those relationships, all of those times...the PAR process has been really good in unpacking some of those as well.(WG2)

It was perceived that the involvement and role of Trial site communities in the national Trial was not initially communicated in a clear or timely manner.

- Why was the mainland announced and [Trial site location] wasn't originally on there? And then we were later added. But it shouldn't have to come out like that. Why not be upfront? This is where we are, this is what we can do. This is what we definitely can't... Where if we just knew straight up this is why we're saying no to what we rather that you know where you stand, what you can work with and what's reasonable. (WG5)

Effective and timely communication was essential to community engagement and managing community expectations. As one WG member described: 
- When it started off, the community engagement, there was a lot of work going on behind the scenes. I'll be frank and say I don't know that we have been on top of communicating our messages as we could to the community... when that happens after there's been a launch in the expectations, people do need regular updates to see what's happening. (WG12)

It was also highlighted that conversations between people not at the frontline of suicide prevention but across sectors, communities, Trial site locations and neighbouring communities were increased as a result of the Trial, highlighting those community members that are well placed to be community "gatekeepers":

- I had an email conversation with [allied health professional] in [coastal town]...she says, "I see suicidal people all the time because of their chronic pain and how it impacts on their life". I'm like, "What do you do with them?" and she's like, "I don't know. I just, like, let them talk". And I'm like, "Do you know there's suicide services who they can go and see for free?" And she's like, "Oh, thank you"...so we're not doing anything new but we're just connecting. (WG1)

For the delivery of some activity types, i.e., training or education, being able to communicate in a way to engage at-risk groups was seen to depend on finding the "right" voice, someone relatable to the cohort, to connect with community members.

- ... the recent activity with Doc Robinson, he's not a professional speaker; he's a dad. Talking about his boy... they [the audience] trusted [him] despite the roughness of the delivery... on their own level... the vast majority of us going out and speaking is not going to reach the people that we talk about as being unreachable. (WG19)

\section{Reaching population groups}

Participants from Working Groups described challenges in reaching at-risk groups, including geographical distances and finding alternative ways to reach the 65+ cohort, who may not use or frequently access emails or social media and still value direct communication via word of mouth as their main source of information.

- One of the barriers with such a widespread rural area ... We have a community that... are an older population, so technology isn't a way...[Host Organisation] uses the radio a lot... (WG25)

- ...in our community, word of mouth seems to be the most effective. (WG4)

Face-to-face activities were the most commonly utilised activity type in the Trial when engaging the male cohort. Taking the training to the priority groups, for example, to their workplace, was suggested as a method to help overcome participation barriers.

- [Hold events] in an environment like a Men's Shed... They come to the Men's Shed because they're isolated, they come there. They don't want to go and see the professionals over there. (E5) 
Engaging senior-level representatives from workplaces with a large number of employees in the priority groups to sit on Working Groups was suggested, to help devise strategies that would increase participation of employees from those organisations.

- It would've been good to have had a HR man from a large workforce...it's all well and good to say, "We'll go out and target male workplaces". Good luck getting the employer to release these people from their paid jobs...backfill...pay other people to come along to training. Like, it sounds good in theory but in practice... (WG1)

\section{The LifeSpan model and activity development}

Participant's perceived LifeSpan to be a helpful starting point for Working Groups and activity focus and development, particularly where there was little experience with suicide prevention activity planning.

- It enabled us to get some activities on the ground...it gave some direction. (WG1)

The model was also appreciated by participants for reflecting the broader areas of people's lives which impact on suicide.

- It seems to encompass all parts of everybody's lives... there's room for help and improvement and support from every area of a person's life, which is really what people need. (WG15)

There was, however, concern across the Working Groups that LifeSpan does not include a postvention strategy as a means to reach those at risk after attempting suicide, as well as those bereaved by suicide who may be at higher risk themselves.

- There was a gap in the model...there's no postvention part of that model that deals with people who have attempted suicide and the people left behind. (WG3)

Participants mentioned that lack of information on how to adapt the framework and accommodate for differences in gender made it challenging for Working Groups to understand how to modify activities and overcome barriers to accessing at-risk groups.

- Gender was, for me, the missing ingredient... that's also historically part of why we haven't really been able to make big inroads on the numbers, because we've not had a gendered focus... programs and services that have been used, or funded in the past, such as Lifeline, tend to reach women better than they do men... we know $75 \%$ of suicide is in men. (E3)

Barriers to implementing the full model centred on its perceived complexity and Working Group members, including the following coordinator, expressed frustration with understanding how the framework should be utilised, particularly with those people who experience health literacy barriers.

- I'm coming from a grassroots level of community development. It was a huge barrier to include people with lived experience, people that have never worked in an office in looking at that model...it is 
extraordinarily difficult for people with low literacy. It's dis-empowering and what you're losing there is some really rough diamonds that have valuable input and linkages to those that are vulnerable. (WG4)

In implementing the Lifespan framework, it was highlighted that co-design with communities and utilising existing resources and relationships was seen as essential for community buy-in and deciding which strategies were to be implemented.

- Sometimes it's not having all pieces of the pie covered...[It's]codesigning with communities to do what communities do best in the context of what resources they have at hand. What relationships do they have? What are their priorities? Rather than sort of tasking them with delivering on the whole of that LifeSpan model. (PHN6)

From a Primary Health Network perspective some strategies, such as those involving emergency and follow up care and using evidence-based treatment, were considered ambitious at the community-level, due to lack of capacity, networks, influence or resources.

- We've asked communities implementing the model to be able to have a significant influence in other areas, like emergency and follow-up care, treatment regimens and even improving safety and access to means... it would take a pretty strategic, well resourced, and capable leadership team to be able to do that...in a short time frame... (PHN6)

Participants highlighted the need for assistance from other organisations, such as Tasmania's Primary Health Network, to address these strategies.

- ...there's a lot of things we'd love to do in [the] health sphere, and accident and emergency and you know, educate GPs and all that kind of stuff. Who in this room can do that? No one... (WG16)

Population group-specific activities often centred on building community capacity and awareness, i.e., the "community engagement" strategy (see Figure 2) and were mainly delivered through a third party to those in gatekeeper roles, rather than the at-risk population themselves.

- A lot of the activities have ended up being one step removed in most cases from the actual person at risk of suicide...it's delivered to maybe a professional or someone else that might have contact with them. (WG14)

\section{Effectiveness and sustainability of activities}

Participants discussed the term "effectiveness", and suggested viewing success along a continuum, and using this to measure outcomes other than suicide rates to reflect progress and what can be learned for future efforts.

- Every relationship that's built, every effort that's made to engage a service is progress. I think use "progress" rather than "success"... It's just the continuum of what you were able to achieve, and then 
understanding the extent to which you were able to achieve what you set out to do...every failure really equals success because this is a trial, it's teaching us something we can apply next time. (PHN6)

- The ultimate measure is the suicide rate, isn't it?...there's so much more you can measure, I mean just raising community awareness about how to talk safely and how to support people...getting a broader community to understand what the impacts of loneliness are... whole community awareness raising. (E2)

Another point of view provided by this participant, was that it was perceived as disconcerting that a primary focus of the Trial was on reducing suicides, but that demonstrating such a reduction likely would not be possible within the life of the Trial in Tasmania.

- I think that was a little bit disconcerting was that- when this was initially introduced, it was with the idea of being able to evaluate the reduction in suicide. (WG12)

Inclusion of people with lived experience within Working Groups was regarded as instrumental in the successful implementation and sustainability of activities.

- Our lived experience group... a partnership that's happened because of the trial... they have really strong stories to tell...it really makes a big difference because the trial may, or may not be there after June but they, the learnings that they have and the confidence that they have, would still be there. (WG2)

This approach was also utilised to reach priority populations and build the capacity of communities, particularly of interest to councils who work at this level.

- What I didn't like was the sense that we were being pushed towards a particular part of the model... around the medical stuff within the hospital system... whereas if we were to work with our strengths as a council, we'd been working more towards the lived experience, towards more community capacity building part. (WG3)

While a focus on activity effectiveness and sustainability were seen as important, there was limited potential to ensure these within activity planning. This was particularly noted at the start of the Trial, with decisions often based on getting anything started, even just one-off activities. Partnerships, continued funding and people's capacity to take ownership were regarded as important elements to sustaining project outcomes.

- A lot of those activities were just a one-off thing. But as we've gone on, we've built on some of those... It's been partnerships, and we're relying on each other to deliver both of our objectives. (WG2)

- Is it sustainable if someone takes ownership of it?...then try find an owner... (WG19)

- We're up to our third event of working together and building those relationships with the Child and Family Centre, with us, with Mission, with the Church Group, with Rotary...that's going to be 
somewhat sustainable going forward, but again, that hinges on funding...those people have a lot of motivation to be involved and do things, but it's hard to do things when you're penny-pinching. (WG1)

Also highlighted was a reflection on the importance of the need for low-cost, high-impact practices for sustainability, i.e., relationship building, using local services, and finding community "ownership" of activities.

- There was probably a conscious idea to work with and embed local services to ... upskill, capacity build... and be involved in sustainability aspects, that's what they're trying to do. (WG12)

- They're fairly simple strategies that don't require a lot of money... build that level of sustainable relationships on the ground... (PHN2)

Sustainability was not always considered in activity planning, with sustainability considered more relevant as the Trial end became nearer.

- [Sustainability is] something that we've struggled with in the past because a lot of those activities were just a one-off thing. As we've gone on, I think we've built on some of those as well, as we've gone on. So it hasn't been a one-off. It's been partnerships and we're relying on each other to deliver both of our objectives. (WG2)

Through training and increased awareness, changes to organisational culture and practices were seen as having a legacy post-Trial, including within population-group specific organisations.

- ... we've been able to train... to educate men in different workplaces... maybe that is an element that then is changing the work culture in those workplaces. (WG1)

- "They're really starting to embed in their practice, as a [workplace], a lot of this work...this is going to continue beyond the pilot, regardless. (WG7)

The host organisation of each Working Group was seen as promoting activity sustainability, with the advantages of drawing on the resources and expertise of host organisations such as local councils.

- Councils run events all the time, it would be very easy for them to put something in those events... They have a lot of levers to pull. Councils know their communities and the different pockets within their communities. (WG1)

It was evident from discussions with community members involved with the Trial that fatigue and despondence from being constantly exposed to suicide meant that any efforts, whether a trial or not, needed to consider lasting impacts for individuals involved, and the communities:

- These communities have built up a level of expectation... trials not continued or converted into more meaningful change, actually supported by dollars- that's three potential [trial site] areas that are going to feel let down, particularly the people that have really been engaged in the process... that's 
always an issue for trials, there's always risks from a design component, like there's uncertainty about what's going to go ahead, you're at risk of losing people because of that... (E2)

Post-Trial, there was a sense that there was a government responsibility for ensuring information would be shared and that continued funding, and support for local communities to build on Trial learnings was important.

- The notion of a trial does suggest ideally that you're going to do something afterward...the 20 something sites around the country potentially all trialling different things...ideally those people should all come together and showcase what they've done, highlight the successes, share what didn't work, you know and sit and acknowledge what didn't work, be encouraged and share that stuff... we shouldn't be having a trial unless there's a recognition or an acknowledgment that we're going to then implement what has worked. Otherwise it just let's communities down. It breaks trust. (E3)

\section{Discussion}

This study explored key stakeholder perceptions around the implementation of a systems-based suicide prevention program, namely the LifeSpan model, in regional and rural communities in Tasmania. Where people were despondent from exposure to suicide within their close-knit communities, the Trial and its associated activities were welcomed. Measures of success were defined by the communities to show the impact of the Trial at this local level, for example improved coordination of existing services, greater awareness of suicide and is prevention. Findings highlighted several perceived limits around establishment of the Trial, the initial limited involvement of key stakeholders, and a lack of role clarity within Working Groups, which impacted activity development and implementation. Rather than strict adherence to the Lifespan model, Working Groups embraced a pragmatic approach of preferring activities that best utilised available resources to meet some of the perceived needs within communities. Activities tended to focus on whole-of-community engagement, awareness raising, and capacity building to build confidence across communities to take action themselves and recognise and respond to members of their communities at-risk.

Barriers were experienced in effectively delivering activities to the priority population groups of men and older aged people, with challenges in both accessing and getting messages across. First, to reach and secure participation of males aged 40-64 who were typically working when activities were offered, a number of strategies were utilised and recommended, including taking training to where men feel comfortable or in familiar settings (for example Men's Sheds, and/or where they work) and helping overcoming stigma at all levels, and the logistical and financial barriers with attending training and activities outside of work hours. The role of people delivering the training was essential to consider, where it has been found that engagement and feelings of connection play a significant part in whether people, however men in particular, access services or supports, and whether they will likely attend again[32]. Additionally, building on previous research exploring barriers to using digital communication and clinical treatment platforms and mobile apps[33, 34], the evaluation has further highlighted that access to, or the 
means to use, digital communication, impacted on engagement of people aged 65 and over. This further highlights the importance of understanding literacy levels with the use of information technology and digital media, and accommodating for these, i.e., through using traditional advertising like TV or mail. Engaging aged care organisations in workplace training and resource distribution needed peak bodies and service providers to be engaged from commencement, further highlighting the need of Primary Health Network-level intervention, to foster these necessary collaborations with senior level management in organisations that are crucial as touchpoints for reaching people most at risk.

It was highlighted that the implementation of the systems model as intended at the community-level needed early, and ongoing, consideration of health literacy early on, and further community engagement and inclusion in key decision making. Alternative approaches and flexibility were essential to ensure most strategies can be/are targeted, particularly those harder to implement strategies such as means restriction. Involving community in these initiatives also builds on the critical point that community are best placed to recognise localised patterns in suicide methods relating to the specific physical spaces within local landscapes[35]. The influence, capacity and resources of organisations such as Tasmania's PHN were required for activities targeting certain strategies, such as those needing to influence primary care systems and increase the capacity of frontline workers to respond to suicidal behaviour. This PHN level involvement will continue to ensure there is a closer approximation of simultaneous implementation with all strategies within a systems approach, as described as being critical for full impact $[9,16,36]$.

Throughout the Trial there were constant requirements for reflecting on what worked well, and not so well, and adapting and improving processes as needed. While a focus on effectiveness and sustainability of activities was seen as important, there was limited potential to ensure these were included for every activity planned, and they instead developed as the Trial evolved. Working Groups described the structures and processes that supported them to deliver activities and how decisions were strongly influenced by the capacity, knowledge, and experiences of community members and people with lived experience involved, and the preferences of their communities. These factors influenced Working Groups decisions to deliver activities only to defined population groups, when it was perceived that the wider community was in just as much need and should not be excluded, a key consideration when working with community-level groups. As previously highlighted $[8,13]$ systems-based approaches have been found promising for suicide prevention, and this study has highlighted the need for community-level insight and consideration with such implementation in regional and rural areas, continued appreciation and utilisation of people with lived experience at all levels, and coordination and support from the PHN level.

\section{Limitations}

Several limitations of the current research should be considered when interpreting the findings. The number of participants varied across the three trial site locations; however data were combined to reflect the overall Tasmanian trial site experience. In addition, processes varied across the Trial site regions, including how Working Groups were structured, their membership and levels of engagement of members, service organisation support, the processes used to recruit service organisations, and the level of

Page 16/24 
community member representation and input. Caution should therefore be exercised when generalising findings across the whole Tasmanian site. Due to a delay between commencement of the Trial and engagement of the evaluation team, and as funding for the evaluation ceased prior to the end of the Trial, stakeholders' perceptions concerning the start and finish of the Trial could not be captured.

\section{Conclusion}

Evaluations of suicide prevention programs in regional, rural, or remote areas are limited, particularly evaluations of programs using systems-based approaches, focusing on men and/or older aged individuals in regional or rural areas of Australia. This evaluation provides a "local-level" perspective which contributes to the evidence of whether, and how, systems-based approaches and the LifeSpan framework in particular, can be implemented in these areas. Considerations for future community-based suicide prevention efforts in regional and rural areas, emphasise the critical role of early community engagement and with people with lived experience, vulnerable population groups, peak bodies, and representation of senior management of service providers. Sustainability of initiatives like the National Suicide Prevention Trial in Tasmania, rely very much on continued community-ownership and involvement, provision of resources, and funding, to support them to take action at the community-level.

\section{Declarations}

\section{Ethics approval and consent to participate}

Ethics approval was granted by the Human Research Ethics Committee (Tasmania) Network (H0017793). All participants provided informed consent to participate.

\section{Consent for publication}

Not applicable.

\section{Availability of data and materials}

The datasets generated and/or analysed during the current study are not publicly available due to the confidential nature of the qualitative data and the ability to recognise individuals, but are available from the corresponding author on reasonable request.

\section{Competing interests}

The authors declare that they have no competing interests.

\section{Funding}

The Tasmanian Suicide Prevention Trial Evaluation was supported and funded by the Tasmanian Primary Health Network under the Primary Health Networks Program - An Australian Government 
Initiative. Tasmania's Primary Health Network were not responsible for any part of the study design, collection, analysis, or interpretation of data, or writing of the manuscript.

\section{Author contributions}

All authors contributed to the conception and design of the work. LG, SA, and TP collected data, LG and SA completed statistical analysis and interpreted the results. LG drafted the first version. TP, SA, JM, and DL contributed to subsequent drafts. All authors approved the final version.

\section{Acknowledgements}

The evaluation team acknowledge and appreciate the input provided by the trial site communities that participated in, and helped lead, the National Suicide Prevention Trial in Tasmania, and those who contributed to this program evaluation.

\section{References}

1. Australian Bureau of Statistics: Causes of Death, Australia, 2020. In: Statistics on the number of deaths, by sex, selected age groups, and cause of death classified to the International Classification of Diseases (ICD). Canberra: Australian Bureau of Statistics; 2021.

2. Australian Bureau of Statistics: Causes of Death, Australia, 2019. In: Statistics on the number of deaths, by sex, selected age groups, and cause of death classified to the International Classification of Diseases (ICD). Canberra: Australian Bureau of Statistics; 2020.

3. Suicide and intentional self-harm [https://www.aihw.gov.au/reports/australias-health/suicide-andintentional-self-harm]

4. Intentional self-harm (suicides), key characteristics, 2019 [https://www.abs.gov.au/statistics/health/causes-death/causes-death-australia/2019\#intentionalself-harm-suicides-key-characteristics]

5. Bartone T, Hickie, I., \& McGorry, P.: COVID-19 Impact Likely to Lead to Increased Rates of Suicide and Mental Illness Joint Statement. In.; 2020.

6. National Suicide Prevention Trial [https://www.lifeinmindaustralia.com.au/programsresources/regional-approaches/phn]

7. Smith L, Purton, T., Auckland, S., Lees, D., \& Mond, J.: Local Evaluation of the Tasmanian Component of the National Suicide Prevention Trial - Preliminary learnings. Australian Journal of Rural and Remote Health 2020, 28:218-223..

8. Sutherland G, Milner A, Dwyer J, Bugeja L, Woodward A, Robinson J, Pirkis J: Implementation and evaluation of the Victorian Suicide Register. Australian and New Zealand journal of public health 2018, 42(3):296-302.

9. Black Dog Institute: Guidance for a Systems Approach to Suicide Prevention for Rural and Remote Communities in Australia. In. Sydney: Black Dog Institute; 2019. 
10. LifeSpan Trials; Evaluation [https://www.blackdoginstitute.org.au/research-centres/lifespantrials/evaluation/]

11. LifeSpan Trials; Outcomes-to-date [https://www.blackdoginstitute.org.au/research-centres/lifespantrials/outcomes-to-date/]

12. Hogan MF, \& Grumet, J. G.: Suicide Prevention: An Emerging Priority For Health Care.. Health Aff (Millwood), 2016, 35(6):1084-1090.

13. Hegerl U, Wittenburg L, Arensman E, Van Audenhove C, Coyne JC, McDaid D, Feltz-Cornelis CMVD, Gusmão R, Kopp M, Maxwell M et al: Optimizing Suicide Prevention Programs and Their Implementation in Europe (OSPI Europe): An evidence-based multi-level approach. BMC public health 2009, 9.

14. Ono $Y$, Awata $S$, lida $H$, Ishida $Y$, Ishizuka $N$, Iwasa $H$, Kamei $Y$, Motohashi $Y$, Nakagawa A, Nakamura $\mathrm{J}$ et al: A community intervention trial of multimodal suicide prevention program in Japan: A Novel multimodal Community Intervention program to prevent suicide and suicide attempt in Japan, NOCOMIT-J. BMC public health 2008, 8.

15. Baker ST, Nicholas J, Shand F, Green R, Christensen H: A comparison of multi-component systems approaches to suicide prevention. Australasian Psychiatry 2018, 26(2):128-131.

16. LifeSpan [https://www.blackdoginstitute.org.au/research/lifespan]

17. Krysinska K, Batterham, P. J., Tye, M., Shand, F., Calear, A. L., Cockayne, N., \& Christensen, H.: Best Strategies for reducing the suicide rate in Australia. Australian and New Zealand Journal of Psychiatry 2016, 50:115-118.

18. Grattidge L, Purton, T., Mond, J., Lees, D., \& Auckland, S.: Participatory Action Research in Suicide Prevention Program Evaluation: Opportunities and Challenges from the National Suicide Prevention Trial, Tasmania. Australian \& New Zealand Journal of Public Health in press.

19. Modified Monash Model [https://www.health.gov.au/health-topics/health-workforce/healthworkforce-classifications/modified-monash-model]

20. Primary Health Tasmania: Suicide prevention trial. Press Release. In.; 2017.

21. Morton KL, Atkin AJ, Corder K, Suhrcke M, Turner D, van Sluijs EMF: Engaging stakeholders and target groups in prioritising a public health intervention: the Creating Active School Environments (CASE) online Delphi study. BMJ open 2017, 7(1):e013340-e013340.

22. Petkovic J, Riddle A, Akl EA, Khabsa J, Lytvyn L, Atwere P, Campbell P, Chalkidou K, Chang SM, Crowe $S$ et al: Protocol for the development of guidance for stakeholder engagement in health and healthcare guideline development and implementation. Systematic Reviews 2020, 9(1):21.

23. Aoun S: Deliberate self-harm in rural Western Australia: results of an intervention study. The Australian and New Zealand journal of mental health nursing 1999, 8(2):65-73.

24. Jones S, Walker C, Miles AC, De Silva E, Zimitat C: A rural, community-based suicide awareness and intervention program. Rural and remote health 2015, 15(1):2972-2972. 
25. Jones M, Ferguson M, Walsh S, Martinez L, Marsh M, Cronin K, Procter N: Perspectives of rural health and human service practitioners following suicide prevention training programme in Australia: A thematic analysis. Health \& social care in the community 2018, 26(3):356-363.

26. Hunt T, Wilson CJ, Woodward A, Caputi P, Wilson I: Intervention among Suicidal Men: Future Directions for Telephone Crisis Support Research. Perspective 2018, 16(1).

27. Perceval M, Reddy P, Ross V, Joiner T, Kolves K: Evaluation of the SCARF Well-Being and Suicide Prevention Program for Rural Australian Communities. J Rural Health 2020, 36(2):247-254.

28. Bantjes J, Swartz L: The Benefits of Robust Debate About the Place of Qualitative Research in Suicide Prevention. Qualitative Health Research 2020, 30(6):944-946.

29. Hjelmeland H, Knizek BL: Why We Need Qualitative Research in Suicidology. Suicide and LifeThreatening Behavior 2010, 40(1):74-80.

30. Lived experience of suicide [https://rosesintheocean.com.au/lived-experience-suicide/]

31. Saunders B, Sim, J., Kingston, T., Baker, S., Waterfield, J., Bartlam, B., Burroughs, H., \& Jinks, C.: Saturation in qualitative research: exploring its conceptualization and operationalization.. Quality and Quantity 2018, 52(4).

32. Seidler ZE, Wilson MJ, Kealy D, Oliffe JL, Ogrodniczuk JS, Rice SM: Men's Dropout From Mental Health Services: Results From a Survey of Australian Men Across the Life Span. American journal of men's health 2021, 15(3):15579883211014776.

33. Pywell J, Vijaykumar S, Dodd A, Coventry L: Barriers to older adults' uptake of mobile-based mental health interventions. DIGITAL HEALTH2020, 6:2055207620905422.

34. Evangelista L, Steinhubl SR, Topol EJ: Digital health care for older adults. The Lancet 2019, 393(10180):1493.

35. Pollock N: Place, the Built Environment, and Means Restriction in Suicide Prevention. International Journal of Environmental Research and Public Health 2019, 16. (4389.).

36. Black Dog Institute: Implementation plan for the systems approach to suicide prevention in NSW: Summary paper. In. Sydney: Black Dog Institute; 2015.

\section{Figures}




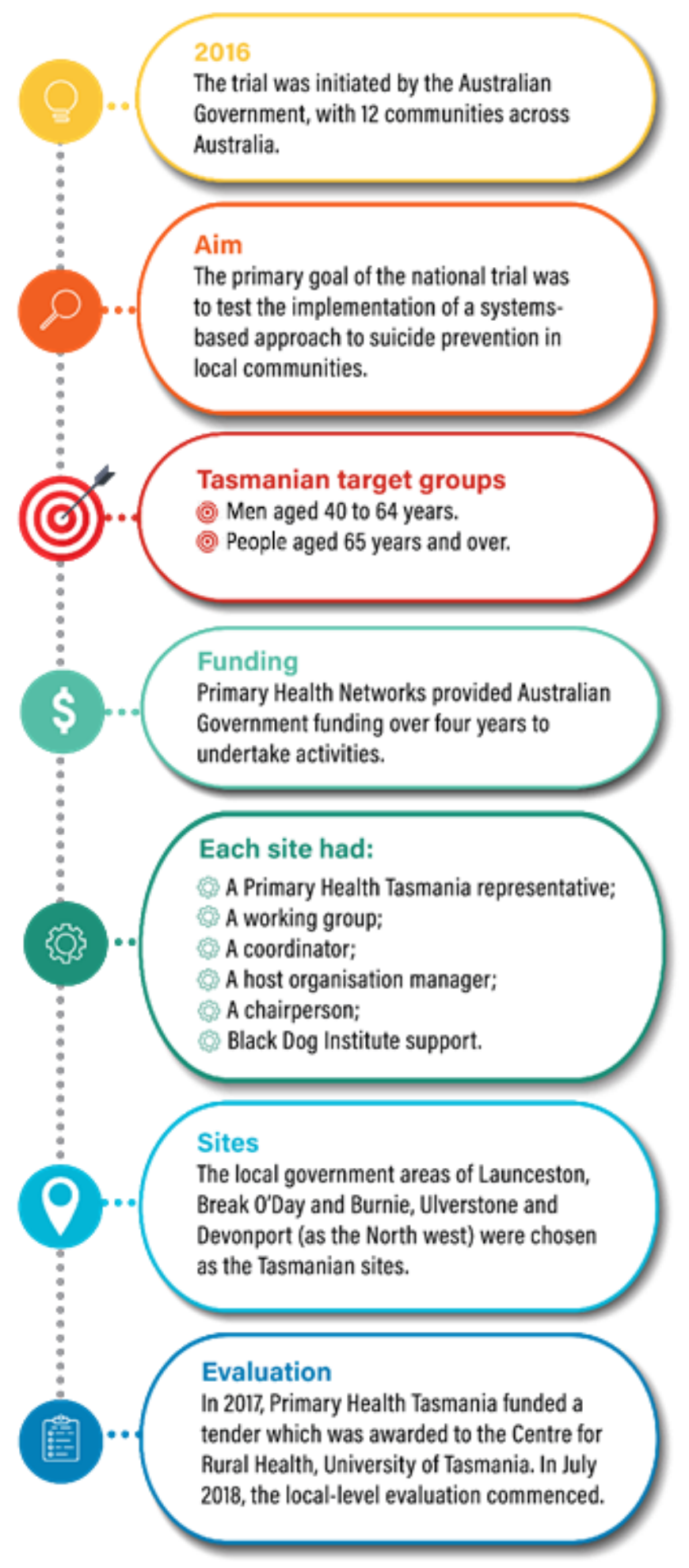

\section{Figure 1}

National Suicide Prevention Trial Tasmania - key background points 


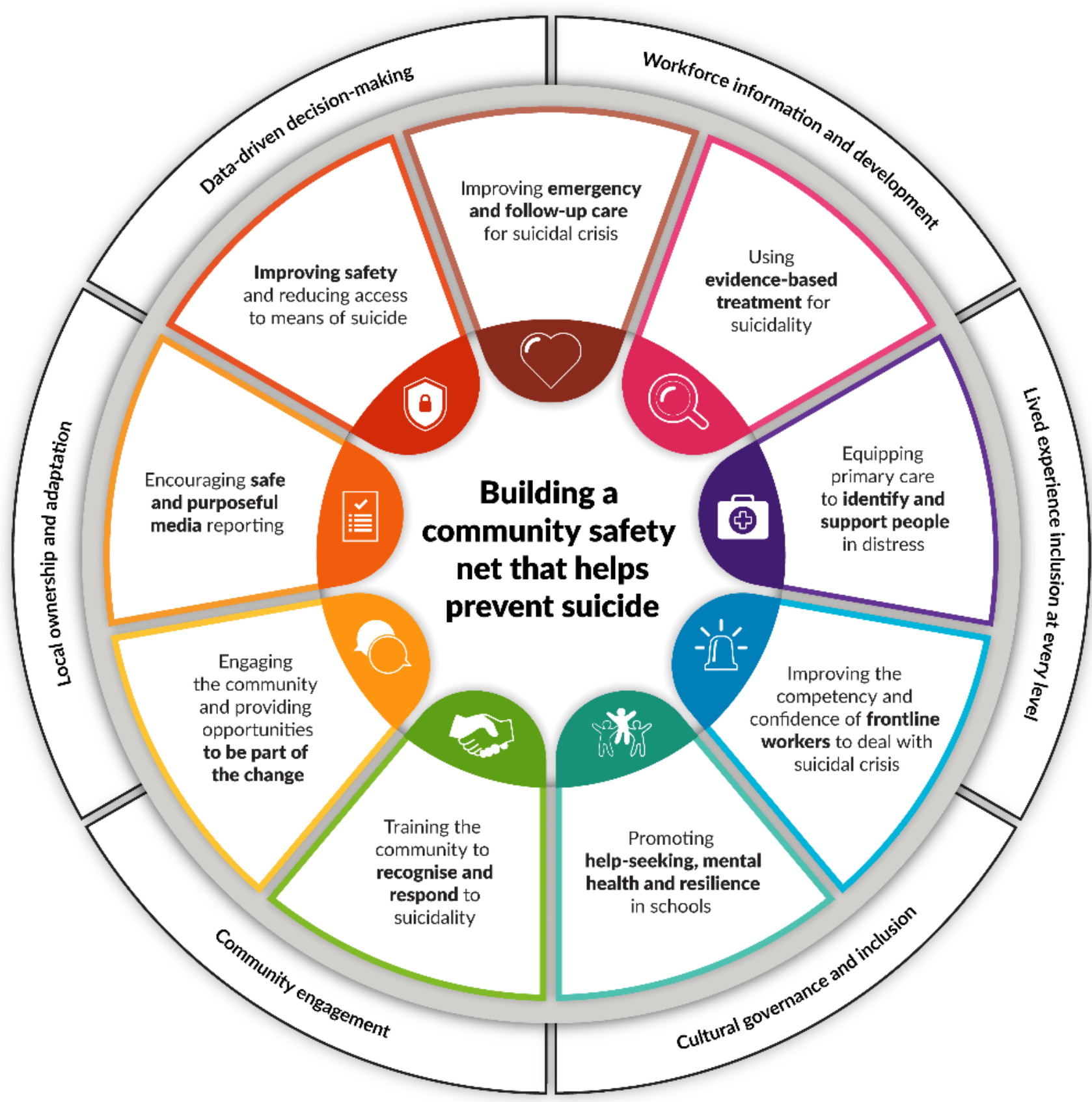

Figure 2

National Suicide Prevention Trial in Tasmania - The LifeSpan Framework Source: LifeSpan model(16) from Black Dog Institute 


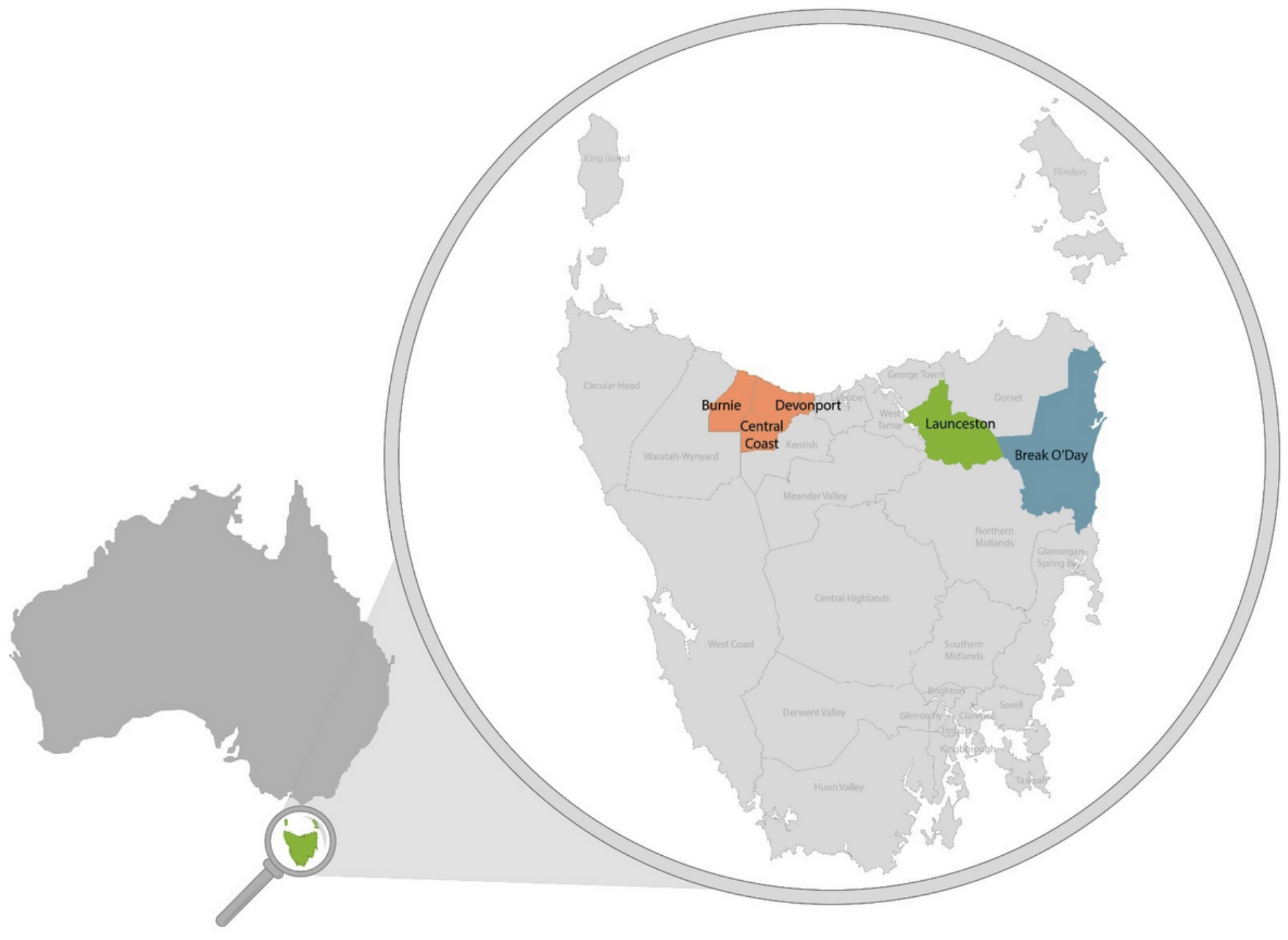

Figure 3

Tasmanian trial site locations -the Northwest coast, Launceston and Break O'Day 
Understanding and Establishing the Trial in

Tasmania

Working Group Governance Structures and

Processes

Communication and Engagement Processes

\section{Reaching Population Groups}

\section{The LifeSpan Model and Activity Development}

\section{Effectiveness and Sustainability of Activities}

\section{Figure 4}

Six primary themes identified from focus group and interview data 